\title{
Periodic and Solitary Wave Solutions of Two Component Zakharov-Yajima-Oikawa System, Using Madelung's Approach ${ }^{\star}$
}

\author{
Anca VISINESCU ${ }^{\dagger}$, Dan GRECU ${ }^{\dagger}$, Renato FEDELE ${ }^{\ddagger}$ and Sergio DE NICOLA $\S$ \\ $\dagger$ Department of Theoretical Physics, National Institute for Physics and Nuclear Engineering, \\ Bucharest, Romania \\ E-mail: avisin@theory.nipne.ro,dgrecu@theory.nipne.ro \\ $\ddagger$ Dipartimento di Scienze Fisiche, Universita Federico II and INFN Sezione di Napoli, \\ Napoli, Italy \\ E-mail: renato.fedele@na.infn.it \\ $\S$ Istituto Nazionale di Ottica del Consiglio Nazionale delle Ricerche, Pozuolli, (Na), Italy \\ E-mail: sergio.denicola@ino.it
}

Received February 10, 2011, in final form April 19, 2011; Published online April 23, 2011

doi:10.3842/SIGMA.2011.041

\begin{abstract}
Using the multiple scales method, the interaction between two bright and one dark solitons is studied. Provided that a long wave-short wave resonance condition is satisfied, the two-component Zakharov-Yajima-Oikawa (ZYO) completely integrable system is obtained. By using a Madelung fluid description, the one-soliton solutions of the corresponding ZYO system are determined. Furthermore, a discussion on the interaction between one bright and two dark solitons is presented. In particular, this problem is reduced to solve a one-component ZYO system in the resonance conditions.
\end{abstract}

Key words: dark-bright solitons; nonlinear Schrödinger equation; Zakharov-Yajima-Oikawa system; Madelung fluid approach

2010 Mathematics Subject Classification: 35Q55; 37K10; 45G15

\section{Introduction}

In many physical applications more than one single wave is propagating in a nonlinear medium, and the interaction of several waves has to be taken into account. In particular, to describe the propagation of three nonlinear pulses in some dispersive material one has to solve simultaneously a set of coupled nonlinear Schrödinger (NLS) equations. For instance, it occurs in: (i) the propagation of solitonlike pulses in birefringent optical fibers [1, 2, 3, 4]; (ii) the nonlinear wave dynamics of Bose-Einstein condensates [5, 6]; (iii) the soliton propagation through optical fiber array $[1,7,8,9]$; (iv) the nonlinear dynamics of gravity waves in crossing sea states [10].

In this paper, a theoretical investigation on the interaction between two bright solitons (short waves) and a dark soliton (long wave) is carried out on the basis of previous investigations that have reduced, under suitable conditions, the study of bright-dark soliton interaction to the study of the existence of a "long wave-short wave resonance" (LW-SW resonance) [11]. This resonance phenomena has quite a large universality. For instance, in plasma physics it describes Langmuir solitons moving near the speed of sound [12, 13, 14], in hydrodynamics it appears in the study of internal gravity waves [15] and a general study of LW-SW resonance [16, 17],

* This paper is a contribution to the Proceedings of the Conference "Symmetries and Integrability of Difference Equations (SIDE-9)" (June 14-18, 2010, Varna, Bulgaria). The full collection is available at http://www.emis.de/journals/SIGMA/SIDE-9.html 
in quasi-one-dimensional molecular crystals it describes the resonance between the exciton and phonon fields in Davydov's model [18, 19, 20, 21]. Recent extensions of the LW-SW resonance to two dimensions and more components have been discussed and solved by several authors $[22,23,24]$.

In the resonance condition, we try to reduce our problem to the study of the completely integrable system of two-component Zakharov-Yajima-Oikawa [12, 13] (see equations (9) and (10) of [11]). Besides its relevance in nonlinear optics, the same system describes the Davydov model with two excitonic components [25]. It is interesting to mention that a vector-like generalization of Zakharov-Benney's equations for long-short wave interaction were obtained 25 years ago in the study of magnon-phonon interaction in a many sublattice XY chain [26], which in the case of one-sublattice XY chain reduces to the Yajima-Oikawa system. In the next section, the basic equations describing the three wave interaction will be presented and, using a multiple scales analysis, the Zakharov-Yajima-Oikawa system is obtained. In Section 3, the Madelung fluid description is used to discuss analytically the above system. In particular, several solitary solutions in the form of traveling waves are presented. In Section 4, the interaction between one-bright and two-dark solitons is discussed, and a simple one-component Zakharov-Yajima-Oikawa system is obtained in resonance condition. In particular, one soliton solution is presented. Finally, remarks and conclusion are presented in Section 5.

\section{Basic equations and multiple scales analysis}

We consider three nonlinear dispersive waves propagating in an optical fiber. We assume that these waves are associated with weakly nonlinear dispersion relations, here denoted by $\omega_{j}=$ $\omega_{j}\left(k_{j}:\left|A_{1}\right|^{2},\left|A_{2}\right|^{2},\left|A_{3}\right|^{2}\right), j=1,2,3$, respectively. Each $A_{j}$ stands for a complex amplitude that, due to the weakly nonlinear dynamics of the medium, is affected by a modulation in both space and time. As it is well known [27], due to this dynamics, each mode of the system can be represented by a wave packet where a carrier wave is amplitude modulated. In order to obtain the evolution equation for each mode, we apply the well-known method of Karpman and Kruskal [28] (see also [27, 29]). To this end, let us denote by $e^{i\left(k_{0} x-\omega_{0} t\right)}$ the basic carrier wave. Then a Taylor expansion around $\left(k_{0}, \omega_{0}\right)$ and $\left|A_{i}\right|=0$ of each $\omega_{i}$ will give

$$
\begin{aligned}
\omega_{i}-\omega_{0}= & \left(\frac{\partial \omega_{i}}{\partial k_{i}}\right)_{0}\left(k_{i}-k_{0}\right)+\frac{1}{2}\left(\frac{\partial^{2} \omega_{i}}{\partial k_{i}^{2}}\right)_{0}\left(k_{i}-k_{0}\right)^{2}+\left(\frac{\partial \omega_{i}}{\partial\left|A_{1}\right|^{2}}\right)_{0}\left|A_{1}\right|^{2} \\
& +\left(\frac{\partial \omega_{i}}{\partial\left|A_{2}\right|^{2}}\right)_{0}\left|A_{2}\right|^{2}+\left(\frac{\partial \omega_{i}}{\partial\left|A_{3}\right|^{2}}\right)_{0}\left|A_{3}\right|^{2}+\cdots
\end{aligned}
$$

Replacing $\omega_{i}-\omega_{0} \simeq-i \frac{\partial}{\partial t}, k_{i}-k_{0} \simeq i \frac{\partial}{\partial x}$, after a translation of coordinate $\left(x \rightarrow x-\left(\frac{\partial \omega_{3}}{\partial k_{3}}\right)_{0} t\right)$, the following nonlinear system of three interacting waves is obtained

$$
\begin{aligned}
& i \frac{\partial A_{1}}{\partial t}+i V_{1} \frac{\partial A_{1}}{\partial x}+\frac{\alpha_{1}}{2} \frac{\partial^{2} A_{1}}{\partial x^{2}}+\alpha_{2}\left|A_{1}\right|^{2} A_{1}+\alpha_{3}\left|A_{2}\right|^{2} A_{1}+\alpha_{4}\left|A_{3}\right|^{2} A_{1}=0, \\
& i \frac{\partial A_{2}}{\partial t}+i V_{2} \frac{\partial A_{2}}{\partial x}+\frac{\beta_{1}}{2} \frac{\partial^{2} A_{2}}{\partial x^{2}}+\beta_{2}\left|A_{1}\right|^{2} A_{2}+\beta_{3}\left|A_{2}\right|^{2} A_{2}+\beta_{4}\left|A_{3}\right|^{2} A_{2}=0 \\
& i \frac{\partial A_{3}}{\partial t}+\frac{\gamma_{1}}{2} \frac{\partial^{2} A_{3}}{\partial x^{2}}+\gamma_{2}\left|A_{1}\right|^{2} A_{3}+\gamma_{3}\left|A_{2}\right|^{2} A_{3}+\gamma_{4}\left|A_{3}\right|^{2} A_{3}=0 .
\end{aligned}
$$

Here we denoted $V_{i}=\left(\frac{\partial \omega_{i}}{\partial k_{i}}\right)_{0}-\left(\frac{\partial \omega_{3}}{\partial k_{3}}\right)_{0}, i=1,2$ and the constants $\alpha_{1}, \beta_{1}, \gamma_{1}$ are related to derivatives of $\omega_{i}$ with respect to $k_{i}$ (ex. $\left.\alpha_{1}=-\left(\frac{\partial^{2} \omega_{1}}{\partial k_{1}^{2}}\right)_{0}, \cdot\right)$ while $\alpha_{2}, \ldots, \gamma_{4}$ to the derivatives with respect to $\left|A_{i}\right|^{2}$ (ex. $\left.\alpha_{2}=\left(\frac{\partial \omega_{1}}{\partial\left|A_{1}\right|^{2}}\right) \cdots\right)$. 
Further on we shall consider channel 3 with normal dispersion and 1 and 2 with the anomalous one [1]. Then following Kivshar [11] it is convenient to introduce new field variables

$$
\begin{aligned}
& A_{1}=\Psi_{1} e^{i \delta_{1} t}, \quad A_{2}=\Psi_{2} e^{i \delta_{2} t}, \quad A_{3}=\left(u_{0}+a(x, t)\right) e^{i(\Gamma t+\phi(x, t))}, \\
& \delta_{i}=\left(\frac{\partial \omega_{i}}{\partial\left|A_{3}\right|^{2}}\right)_{0} u_{0}^{2}, \quad \Gamma=\left(\frac{\partial \omega_{3}}{\partial\left|A_{3}\right|^{2}}\right)_{0} u_{0}^{2}
\end{aligned}
$$

$\left(u_{0}, a(x, t), \phi(x, t)\right.$ being real quantities). Note that for solitary wave solutions the additional conditions of vanishing of $a$ at the infinity has to be imposed. Then the equations of $A_{1}$ and $A_{2}$ become, respectively

$$
\begin{aligned}
& i \frac{\partial \Psi_{1}}{\partial t}+i V_{1} \frac{\partial \Psi_{1}}{\partial x}+\frac{\alpha_{1}}{2} \frac{\partial^{2} \Psi_{1}}{\partial x^{2}}+\left(\alpha_{2}\left|\Psi_{1}\right|^{2}+\alpha_{3}\left|\Psi_{2}\right|^{2}\right) \Psi_{1}+2 \alpha_{4} u_{0} a \Psi_{1}+\alpha_{4} a^{2} \Psi_{1}=0, \\
& i \frac{\partial \Psi_{2}}{\partial t}+i V_{2} \frac{\partial \Psi_{2}}{\partial x}+\frac{\beta_{1}}{2} \frac{\partial^{2} \Psi_{2}}{\partial x^{2}}+\left(\beta_{2}\left|\Psi_{1}\right|^{2}+\beta_{3}\left|\Psi_{2}\right|^{2}\right) \Psi_{2}+2 \beta_{4} u_{0} a \Psi_{2}+\beta_{4} a^{2} \Psi_{2}=0 .
\end{aligned}
$$

As concerns the $A_{3}$-equation, separating the real and the imaginary part, the following system of coupled equations is obtained

$$
\begin{aligned}
& \frac{\partial a}{\partial t}+\frac{\gamma_{1}}{2} u_{0} \frac{\partial^{2} \phi}{\partial x^{2}}+(\text { nonlinear terms })=0 \\
& -\frac{\partial \phi}{\partial t}+2 \gamma_{4} u_{0} a+\frac{\gamma_{1}}{2 u_{0}} \frac{\partial^{2} a}{\partial x^{2}}+\left(\gamma_{2}\left|\Psi_{1}\right|^{2}+\gamma_{3}\left|\Psi_{2}\right|^{2}\right)+(\text { nonlinear terms })=0 .
\end{aligned}
$$

In both these equations the parenthesis $(\cdots)$ contains all the other nonlinear terms which will be irrelevant in a multiple scales analysis. From these last two equations the following equation satisfied by $a(x, t)$ is easily obtained

$$
\begin{aligned}
\frac{\partial^{2} a}{\partial t^{2}} & +\gamma_{1} \gamma_{4} u_{0}^{2} \frac{\partial^{2} a}{\partial x^{2}}+\frac{\gamma_{1}^{2}}{4} \frac{\partial^{4} a}{\partial x^{4}}+u_{0} \frac{\gamma_{1}}{2} \frac{\partial^{2}}{\partial x^{2}}\left(\gamma_{2}\left|\Psi_{1}\right|^{2}+\gamma_{3}\left|\Psi_{2}\right|^{2}\right) \\
& + \text { (higher order nonlinear terms in }(a, \phi) \text { and their derivatives })=0 .
\end{aligned}
$$

The linear part of the $a$ equation corresponds to an acoustic field with dispersion relation $\left(\gamma_{1}<0, \gamma_{4}>0\right)$

$$
\omega=c k \sqrt{1+\frac{\gamma_{1}^{2}}{4 c^{2}} k^{2}} \simeq c k\left(1+\frac{\gamma_{1}^{2}}{8 c^{2}} k^{2}\right)
$$

and phase velocity $c=\omega / k$, where $c^{2}=\left|\gamma_{1}\right| \gamma_{4} u_{0}^{2}$.

We shall perform a multiple scales analysis of the system $(2)+(3)$ [11]. We introduce new scaled variables

$$
t \Rightarrow \epsilon t, \quad x \Rightarrow \sqrt{\epsilon}(x-c t)
$$

and new functions

$$
a \Rightarrow \epsilon a, \quad \phi \Rightarrow \epsilon \phi, \quad \Psi_{1} \Rightarrow \epsilon^{\frac{3}{4}} \Psi_{1}, \quad \Psi_{2} \Rightarrow \epsilon^{\frac{3}{4}} \Psi_{2} .
$$

Then in order $\frac{5}{2}$ in $\epsilon$ from $a$ equation we obtain

$$
-2 c \frac{\partial a}{\partial t}+u_{0} \frac{\gamma_{1}}{2} \frac{\partial}{\partial x}\left(\gamma_{2}\left|\Psi_{1}\right|^{2}+\gamma_{3}\left|\Psi_{2}\right|^{2}\right)=0
$$

All the other terms in $a$ equation contribute to higher order in $\epsilon$. In the order $\frac{5}{4}$ from $\Psi_{i}$ equations we obtain $V_{1}=V_{2}=c$. This is the well known long wave-short wave (LW-SW) 
resonance condition: "the group velocity $V$ of the $S W$ is equal to the phase velocity of the $L W^{\prime}$, [16]. In the next order $\left(\frac{7}{4}\right)$ in $\epsilon$ from the $\Psi$ equations we get

$$
\begin{aligned}
& i \frac{\partial \Psi_{1}}{\partial t}+\frac{\alpha_{1}}{2} \frac{\partial^{2} \Psi_{1}}{\partial x^{2}}+2 \alpha_{4} u_{0} a \Psi_{1}=0 \\
& i \frac{\partial \Psi_{2}}{\partial t}+\frac{\beta_{1}}{2} \frac{\partial^{2} \Psi_{2}}{\partial x^{2}}+2 \beta_{4} u_{0} a \Psi_{2}=0
\end{aligned}
$$

The equations $(4)+(5)$ represent an 1-dimensional 2-components Zakharov [12], YajimaOikawa [13] system. As mentioned in the Introduction the same system in the same LW-SW resonance condition was obtained in a Davydov model with two excitonic modes coupled with a phonon field [25]. The same line of reasoning was used in [24] for three interacting waves in 2-dimensions.

\section{Madelung fluid description}

The special case $\left(\alpha_{i}=\beta_{i}, \gamma_{2}=\gamma_{3}\right)$ is completely integrable [24] and will be considered in the following. In this case, simplifying the notations, the system $(4)+(5)$ is written in the following form $(\gamma>0, \beta>0)$

$$
\begin{aligned}
& \frac{\partial a}{\partial t}-\gamma \frac{\partial}{\partial x}\left(\left|\Psi_{1}\right|^{2}+\left|\Psi_{2}\right|^{2}\right)=0, \\
& i \frac{\partial \Psi_{i}}{\partial t}+\frac{1}{2} \frac{\partial^{2} \Psi_{i}}{\partial x^{2}}-\beta \Psi_{i} a=0, \quad i=1,2 .
\end{aligned}
$$

The $\Psi_{i}$ equations will be transformed using a Madelung fluid description [30, 31]. We write

$$
\Psi_{i}=\sqrt{\rho_{i}} e^{i \theta_{i}}
$$

where $\rho_{i}, \theta_{i}$ are real functions of $(x, t)$ and moreover $\rho_{i}$ are positive quantities. Introducing this expression into $a$-equation this becomes

$$
\frac{\partial a}{\partial t}-\gamma \frac{\partial}{\partial x}\left(\rho_{1}+\rho_{2}\right)=0
$$

while from the $\Psi_{i}$ equations, after the separation of real and imaginary parts, we obtain

$$
\frac{\partial \rho_{i}}{\partial t}+\frac{\partial}{\partial x}\left(v_{i} \rho_{i}\right)=0
$$

which is a continuity equation for the fluid densities $\rho_{i}=\left|\Psi_{i}\right|^{2}$ with $v_{i}(x, t)=\frac{\partial \theta_{i}(x, t)}{\partial x}$ the fluid velocities components and

$$
-\frac{\partial \theta_{i}}{\partial t}+\frac{1}{2} \frac{1}{\sqrt{\rho_{i}}} \frac{\partial^{2} \sqrt{\rho_{i}}}{\partial x^{2}}-\frac{1}{2}\left(\frac{\partial \theta_{i}}{\partial x}\right)^{2}-\beta a=0 .
$$

Differentiating this last expression with respect to $x$ the following equations of motion for the fluid velocities $v_{i}$ are obtained

$$
\left(\frac{\partial}{\partial t}+v_{i} \frac{\partial}{\partial x}\right) v_{i}=\frac{1}{2} \frac{\partial}{\partial x}\left(\frac{1}{\sqrt{\rho_{i}}} \frac{\partial^{2} \sqrt{\rho_{i}}}{\partial x^{2}}\right)-\beta \frac{\partial a}{\partial x} .
$$

In the right hand side of (8) $a(x, t)$ plays the role of external potential, and the first term is the derivative of the so called Bohm potential, $\frac{1}{2} \frac{1}{\sqrt{\rho_{i}}} \frac{\partial^{2} \sqrt{\rho_{i}}}{\partial x^{2}}$, and contains all the diffraction effects 
(quantum effects in quantum problems). By a series of transformations the equation (8) is written as [31]

$$
-\rho_{i} \frac{\partial v_{i}}{\partial t}+v_{i} \frac{\partial \rho_{i}}{\partial t}+2\left[c_{i}(t)-\int \frac{\partial v_{i}}{\partial t} d x\right] \frac{\partial \rho_{i}}{\partial x}+\frac{1}{4} \frac{\partial^{3} \rho_{i}}{\partial x^{3}}-\beta \rho_{i} \frac{\partial}{\partial x} a-2 \beta a \frac{\partial \rho_{i}}{\partial x}=0,
$$

where $c_{i}$ are arbitrary integration constants with respect to $x$, eventually time dependent. Although (9) seems to be more complicated then the initial equation (7), it can be solved in two special situations, namely

- motion with constant velocities $v_{1}=v_{2}=v_{0}$,

- motion with stationary profile current velocity, when all the quantities $\rho_{i}(x, t), v_{i}(x, t)$, $a(x, t)$ are depending on $x$ and $t$ through the combination $\xi=x-u_{0} t$.

Both cases will be analyzed in the following.

\subsection{Motion with constant velocity $\left(v_{1}=v_{2}=v_{0}\right)$}

In this case from the continuity equations (6) one sees that both $\rho_{1}(x, t)$ and $\rho_{2}(x, t)$ depend on $\xi=x-v_{0} t$. We assume that also $a(x, t)$ depends only on $\xi$. Then the $a$ equation gives

$$
a=-\mu\left(\rho_{1}+\rho_{2}\right), \quad \mu=\frac{\gamma}{v_{0}}
$$

and the equations (9) write

$$
\frac{1}{4} \frac{d^{3} \rho_{i}}{d \xi^{3}}-E_{i} \frac{d \rho_{i}}{d \xi}+2 \mu\left(\rho_{1}+\rho_{2}\right) \frac{d \rho_{i}}{d \xi}+\mu \rho_{i} \frac{d}{d \xi}\left(\rho_{1}+\rho_{2}\right)=0,
$$

where by $E_{i}$ we denoted $-\left(2 c_{i}-v_{0}^{2}\right), \beta \mu \rightarrow \mu$. We shall discuss firstly the situation $E_{1}=E_{2}$, the discussion of the more general case $E_{1} \neq E_{2}$ being postponed for the next subsection. Then the equations (11) becomes

$$
\frac{1}{4} \frac{d^{3} \rho_{i}}{d \xi^{3}}-E \frac{d \rho_{i}}{d \xi}+\mu \rho_{i} \frac{d}{d \xi}\left(\rho_{1}+\rho_{2}\right)+2 \mu\left(\rho_{1}+\rho_{2}\right) \frac{d \rho_{i}}{d \xi}=0 .
$$

These are exactly the equations obtained in the case of Manakov's model [32] and extensively discussed by us in $[33,34]$. In the following we shall present several periodic and traveling wave solutions of (12).

It is convenient to introduce the quantities $z_{+}=\rho_{1}+\rho_{2}$, and $z_{-}=\rho_{1}-\rho_{2}$; they satisfy the following equations

$$
\begin{aligned}
& \frac{1}{4} \frac{d^{3} z_{+}}{d \xi^{3}}-E \frac{d z_{+}}{d \xi}+\frac{3}{2} \mu \frac{d z_{+}^{2}}{d \xi}=0 \\
& \frac{1}{4} \frac{d^{3} z_{-}}{d \xi^{3}}-E \frac{d z_{-}}{d \xi}+\mu z_{-} \frac{d z_{+}}{d \xi}+2 \mu z_{+} \frac{d z_{-}}{d \xi}=0
\end{aligned}
$$

The second equation is a linear differential equation for $z_{-}$once $z_{+}$is known. A special solution is

$$
z_{-}=\left(p_{1}^{2}-p_{2}^{2}\right) z_{+}, \quad p_{1}^{2}+p_{2}^{2}=1,
$$

which together with the definition of $z_{+}$gives

$$
\rho_{1}=p_{1}^{2} z_{+}, \quad \rho_{2}=p_{2}^{2} z_{+}
$$


and the problem is reduced in this simple case to find a solution of $z_{+}$-equation. This integrated twice gives

$$
\frac{1}{4}\left(\frac{d z_{+}}{d \xi}\right)^{2}=-\mu z_{+}^{3}+E z_{+}^{2}+A z_{+}+B=P_{3}\left(z_{+}\right),
$$

where $P_{3}\left(z_{+}\right)$is a third order polynomial in $z_{+}$. The periodic solution of this equation are easily expressed through Jacobi elliptic functions.

For constant velocities, as it is easily seen from (8), the densities $\rho_{i}$ have to satisfy the additional conditions

$$
\frac{1}{2} \frac{1}{\sqrt{\rho_{i}}} \frac{\partial^{2} \sqrt{\rho_{i}}}{\partial x^{2}}+\mu z_{+}(\xi)=\lambda_{i}
$$

which for the previous solutions are satisfied if $\lambda=E / 2$ and $B=0$.

Now let us assume that the third order polynomial $P_{3}\left(z_{+}\right)$has three distinct roots

$$
P_{3}\left(z_{+}\right)=-\mu\left(z_{+}-z_{1}\right)\left(z_{+}-z_{2}\right)\left(z_{+}-z_{3}\right) .
$$

The restriction $B=0$ means that one of the roots $z_{2}$ or $z_{3}$ is zero. We are interested in positive solutions of (13) for which $P_{3}\left(z_{+}\right)$is also positive. The periodic solutions of (14) can be expressed through Jacobi elliptic functions and taking into account the positivity requirement mentioned before we identify two acceptable situations [35]

$$
\begin{aligned}
& z_{1}>0, \quad z_{2}=0, \quad z_{3}<0, \quad z_{+}=z_{1} \mathrm{cn}^{2} u, \\
& u=\frac{2 \sqrt{\mu}}{g} \xi, \quad k^{2}=\frac{z_{1}}{z_{1}+\left|z_{3}\right|}, \quad g=\frac{2}{\sqrt{z_{1}+\left|z_{3}\right|}}
\end{aligned}
$$

and

$$
\begin{aligned}
& z_{3}=0, \quad 0<z_{2}<z_{1}, \quad z_{+}=z_{1}-\left(z_{1}-z_{2}\right) \operatorname{sn}^{2} u \\
& u=\frac{2 \sqrt{\mu}}{g} \xi, \quad k^{2}=\frac{z_{1}-z_{2}}{z_{1}}, \quad g=\frac{2}{\sqrt{z_{1}}} .
\end{aligned}
$$

Solitary wave solutions are obtained in the limiting case $k=1$ when $\operatorname{cn} u \rightarrow \operatorname{sech} u$, sn $u \rightarrow$ tanh $u$, and both solutions (15) and (16) become a bright soliton

$$
z_{+} \rightarrow z_{1} \frac{1}{\cosh ^{2} u}, \quad u=\frac{2 \sqrt{\mu}}{g}, \quad g=\frac{2}{\sqrt{z_{1}}} .
$$

It is clear that in this case no energy transfer between the two components takes place.

The phase $\theta(x, t)$ is easily calculated writing $\theta_{i}(x, t)=v_{0} x+\gamma_{i}(t)$; then using $(7)$ we get

$$
\theta_{i}=v_{0} x-\left(\frac{1}{2} v_{0}^{2}-\frac{E}{2}\right) t+\delta_{i}
$$

As far as the field $a$ is concerned, the solitary wave solution is

$$
a(u)=-\frac{\mu z_{1}}{\cosh ^{2} u}
$$

and, for $\mu z_{1} \leq u_{0}$, the field $\Psi_{3}$ describes a grey solution (the inequality gives the dark solution). 


\subsection{Motion with stationary-profile current velocity}

In the case when all the functions depend only on $\xi=x-u_{0} t$ integrating the continuity equation (6) we get

$$
v_{i}(x, t)=u_{0}+\frac{A_{i}}{\rho_{i}}
$$

with $A_{i}$ some integration constants. It is easily seen that the equations of motion keep the same form as (11) with $E_{i}=-\left(2 c_{i}+u_{0}^{2}\right)$.

For $E_{1}=E_{2}$, the results are almost the same as those of the previous subsection.

However, for $E_{1} \neq E_{2}$, the system (11) will be solved by using a direct method. To this end, we look for solutions of the form

$$
\rho_{i}=A_{i}+B_{i} \operatorname{sn} u, \quad u=2 \lambda \xi,
$$

with $A_{i}, B_{i}, \lambda$ constants to be determined. Introducing into (11) we get [33]

$$
\begin{aligned}
& B_{1}+B_{2}=-\frac{4 \lambda^{2} k^{2}}{\mu}, \\
& -\left[4 \lambda^{2}\left(1+k^{2}\right)+E_{i}\right] B_{i}+\mu\left(B_{1}+B_{2}\right) A_{i}+2 \mu\left(A_{1}+a_{2}\right) B_{i}=0 .
\end{aligned}
$$

Defining the following new quantities $a_{i}, b_{i}, e_{0}$ and $\delta$, i.e.,

$$
\begin{aligned}
& B_{i}=-\frac{4 \lambda^{2} k^{2}}{\mu} b_{i}, \quad A_{i}=\frac{4 \lambda^{2} k^{2}}{\mu} a_{i}, \\
& E_{1}=4 \lambda^{2} k^{2}\left(e_{0}+\delta\right), \quad E_{2}=4 \lambda^{2} k^{2}\left(e_{0}-\delta\right), \quad \delta>0
\end{aligned}
$$

the first equation (17) gives

$$
b_{1}+b_{2}=1 \text {, }
$$

while from the second, after a little algebra, we get

$$
\begin{aligned}
& a_{1}=\frac{1}{3}\left(e_{0}+\frac{1+k^{2}}{k^{2}}+\delta+4 \delta\left(1-b_{1}\right)\right) b_{1}, \\
& a_{2}=\frac{1}{3}\left(e_{0}+\frac{1+k^{2}}{k^{2}}-\delta-4 \delta\left(1-b_{2}\right)\right) b_{2} .
\end{aligned}
$$

As it is expected this result verify the symmetry condition $1 \leftrightarrow 2$ if $\delta \leftrightarrow-\delta$.

Several restrictions result from the positiveness of $\rho_{i}$. If both $b_{i}$ are positive quantities smaller than unity this requirement implies

$$
a_{i}>b_{i}>0 \text {. }
$$

Introducing the notation

$$
p=\frac{1}{3}\left(e+\frac{1+k^{2}}{k^{2}}-5 \delta\right)
$$

the condition (19) is satisfied if $p>1$. In the limiting case $k^{2}=1$ the solutions are

$$
\rho_{1}=\frac{4 \lambda^{2}}{\mu}\left(a_{1}-b_{1} \tanh ^{2} u\right), \quad \rho_{2}=\frac{4 \lambda^{2}}{\mu}\left(a_{2}-b_{2} \tanh ^{2} u\right)
$$

representing shifted bright solitons.

We can now calculate the field $a$ from (10) and using the limit $a(u) \rightarrow 0$ as $u \rightarrow \infty$, we get $a_{1}+a_{2}=1$. Furthermore, making use of (18), this condition becomes

$$
b_{1}-b_{2}=\frac{1}{\delta}\left(2-e_{0}-\frac{1}{k^{2}}\right),
$$

where we have used the condition $b_{1}+b_{2}=1$. 


\section{One bright-two dark solitons interaction}

The previous discussion is easily extended to the situation when two waves have normal dispersion and one is anomalous, corresponding to one bright-two dark solitons interaction. Such vector solitons of mixed bright-dark types are of interest in quasi-one-dimensional Bose-Einstein condensates (see [6] and references therein). We are interested in a SW-LW resonance regime, so several restrictions on the model of three interacting waves will be imposed. With the notations of section 2 we shall assume $\left(\frac{\partial \omega_{2}}{\partial k_{2}}\right)_{0}=\left(\frac{\partial \omega_{3}}{\partial k_{3}}\right)_{0}$ and after the corresponding translation of coordinates instead of system (1) we get

$$
\begin{aligned}
& i \frac{\partial A_{1}}{\partial t}+i v \frac{\partial A_{1}}{\partial x}+\frac{\alpha_{1}}{2} \frac{\partial^{2} A_{1}}{\partial x^{2}}+\alpha_{2}\left|A_{1}\right|^{2} A_{1}+\alpha_{3}\left|A_{2}\right|^{2} A_{1}+\alpha_{4}\left|A_{3}\right|^{2} A_{1}=0, \\
& i \frac{\partial A_{2}}{\partial t}+\frac{\beta_{1}}{2} \frac{\partial^{2} A_{2}}{\partial x^{2}}+\beta_{2}\left|A_{1}\right|^{2} A_{2}+\beta_{3}\left|A_{2}\right|^{2} A_{2}+\beta_{4}\left|A_{3}\right|^{2} A_{3}=0, \\
& i \frac{\partial A_{3}}{\partial t}+\frac{\gamma_{1}}{2} \frac{\partial^{2} A_{3}}{\partial x^{2}}+\gamma_{2}\left|A_{1}\right|^{2} A_{3}+\gamma_{3}\left|A_{2}\right|^{2} A_{3}+\gamma_{4}\left|A_{3}\right|^{2} A_{3}=0,
\end{aligned}
$$

where $v=\left(\frac{\partial \omega_{1}}{\partial k_{1}}\right)_{0}-\left(\frac{\partial \omega_{2}}{\partial k_{2}}\right)_{0}$ and the rest of the notations are the same as in Section 2.

Further on we shall consider the channels 2 and 3 with normal dispersion and the channel 1 with anomalous one and introduce new field variables by

$$
A_{1}=\Psi e^{i \delta t}, \quad A_{2}=\left(u_{2}+a_{2}(x, t)\right) e^{i\left(\Gamma_{2} t+\phi_{2}(x, t)\right)}, \quad A_{3}=\left(u_{3}+a_{3}(x, t)\right) e^{i\left(\Gamma_{3} t+\phi_{3}(x, t)\right)}
$$

with $u_{2}, u_{3}, a_{2}(x, t), a_{3}(x, t), \phi_{2}(x, t), \phi_{3}(x, t)$ real quantities, and

$$
\delta=\alpha_{3} u_{2}^{2}+\alpha_{4} u_{3}^{2}, \quad \Gamma_{2}=\beta_{3} u_{2}^{2}+\beta_{4} u_{3}^{2}, \quad \Gamma_{3}=\gamma_{3} u_{2}^{2}+\gamma_{4} u_{3}^{2} .
$$

The $A_{1}$-equation transforms into

$$
i \frac{\partial \Psi}{\partial t}+i v \frac{\partial \Psi}{\partial x}+\frac{\alpha_{1}}{2} \frac{\partial^{2} \Psi}{\partial x^{2}}+\alpha_{2}|\Psi|^{2} \Psi+2\left(\alpha_{3} u_{2} a_{2}+\alpha_{4} u_{3} a_{3}\right) \Psi+\left(\alpha_{3} a_{2}^{2}+\alpha_{4} a_{3}^{2}\right) \Psi=0 .
$$

To get the relevant equations for $a_{2}, a_{3}$ we have proceed like in Section 2, obtaining the following equations for the amplitudes $a_{2}, a_{3}$, i.e.,

$$
\begin{aligned}
& \frac{\partial^{2} a_{2}}{\partial t^{2}}+\frac{\beta_{1}}{2} u_{2} \frac{\partial^{2}|\Psi|^{2}}{\partial x^{2}}+\beta_{1} u_{2}\left(\beta_{3} u_{2} \frac{\partial^{2} a_{1}}{\partial x^{2}}+\beta_{4} u_{3} \frac{\partial^{2} a_{3}}{\partial x^{2}}\right)+\frac{\beta_{1}^{2}}{4} \frac{\partial^{4} a_{2}}{\partial x^{4}}+(\text { nl. terms })=0 \\
& \frac{\partial^{2} a_{3}}{\partial t^{2}}+\frac{\gamma_{1}}{2} u_{3} \frac{\partial^{2}|\Psi|^{2}}{\partial x^{2}}+\gamma_{1} u_{3}\left(\gamma_{3} u_{2} \frac{\partial^{2} a_{2}}{\partial x^{2}}+\gamma_{4} u_{3} \frac{\partial^{2} a_{3}}{\partial x^{2}}\right)+\frac{\gamma_{1}^{2}}{4} \frac{\partial^{4} a_{3}}{\partial x^{4}}+(\text { nl. terms })=0
\end{aligned}
$$

where, as mentioned before the parenthesis $(\cdots)$ of each equation group all the nonlinear terms which will be irrelevant in a multiple scales analysis. Furthermore we shall consider only the special case $u_{2}=u_{3}=u_{0}, \alpha_{2}=\alpha_{3}, \beta_{2}=\beta_{3}, \gamma_{2}=\gamma_{3}$ and neglect the last nonlinear terms in (20) and all the parenthesis in (21), remaining with the following system of coupled equations

$$
\begin{aligned}
& i \frac{\partial \Psi}{\partial t}+i v \frac{\partial \Psi}{\partial x}+\frac{\alpha_{1}}{2} \frac{\partial^{2} \Psi}{\partial x^{2}}+\alpha_{2}|\Psi|^{2} \Psi+2 u_{0} \alpha_{3}\left(a_{2}+a_{3}\right) \Psi=0 \\
& \frac{\partial^{2} a_{2}}{\partial t^{2}}+\frac{\beta_{1}}{2} u_{0} \frac{\partial^{2}|\Psi|^{2}}{\partial x^{2}}+\beta_{1} \beta_{3} u_{0}^{2} \frac{\partial^{2}}{\partial x^{2}}\left(a_{2}+a_{3}\right)+\frac{\beta_{1}^{2}}{4} \frac{\partial^{4} a_{2}}{\partial x^{2}}=0 \\
& \frac{\partial^{2} a_{2}}{\partial t^{2}}+\frac{\gamma_{1}}{2} u_{0} \frac{\partial^{2}|\Psi|^{2}}{\partial x^{2}}+\gamma_{1} \gamma_{3} u_{0}^{2} \frac{\partial^{2}}{\partial x^{2}}\left(a_{2}+a_{3}\right)+\frac{\gamma_{1}^{2}}{4} \frac{\partial^{4} a_{3}}{\partial x^{2}}=0 .
\end{aligned}
$$


As in $\Psi$-equation appears the combination $a_{+}=a_{2}+a_{3}$ it is convenient to add the $a_{2^{-}}$and $a_{3}$-equations. In a multiple scales analysis the fourth order derivatives will contribute to higher order so we can drop them at the present stage. Then the system (22) reduces to

$$
\begin{aligned}
& i \frac{\partial \Psi}{\partial t}+i v \frac{\partial \Psi}{\partial x}+\frac{\alpha_{1}}{2} \frac{\partial^{2} \Psi}{\partial x^{2}}+\alpha_{2}|\Psi|^{2} \Psi+2 u_{0} \alpha_{3} a_{+} \Psi=0, \\
& \frac{\partial^{2} a_{+}}{\partial t^{2}}+\frac{u_{0}}{2}\left(\beta_{1}+\gamma_{1}\right) \frac{\partial^{2}|\Psi|^{2}}{\partial x^{2}}+u_{0}^{2}\left(\beta_{1} \beta_{3}+\gamma_{1} \gamma_{3}\right) \frac{\partial^{2} a_{+}}{\partial x^{2}}=0 .
\end{aligned}
$$

In the resonance condition $\left(\beta_{1}<0, \gamma_{1}<0\right)$

$$
v=c, \quad c^{2}=u_{0}^{2}\left(\left|\beta_{1}\right| \beta_{3}+\left|\gamma_{1}\right| \gamma_{3}\right)
$$

and by using the multiple scales analysis

$$
x \rightarrow \sqrt{\epsilon}(x-c t), \quad t \rightarrow \epsilon t, \quad a_{+} \rightarrow \epsilon a_{+}, \quad \Psi \rightarrow \epsilon^{\frac{3}{4}} \Psi
$$

the system (23) becomes the well known one-component ZYO system

$$
\begin{aligned}
& i \frac{\partial \Psi}{\partial t}+\frac{\alpha_{1}}{2} \frac{\partial^{2} \Psi}{\partial x^{2}}+2 u_{0} \alpha_{3} a_{+} \Psi=0, \\
& \frac{\partial a_{+}}{\partial t}+\frac{u_{0}}{4 c}\left(\left|\beta_{1}\right|+\left|\gamma_{1}\right|\right) \frac{\partial|\Psi|^{2}}{\partial x}=0 .
\end{aligned}
$$

The one-soliton solution of (24) is given by

$$
a_{+}=M \operatorname{sech}^{2} \theta, \quad \Psi=N e^{i \phi} \operatorname{sech} \theta, \quad \theta=\mu\left(x-x_{0}\right)-\nu t, \quad \phi=\xi x-\eta t,
$$

where

$$
M=\frac{\alpha}{\beta} \mu^{2}, \quad N^{2}=\frac{\alpha}{\beta \gamma} \mu \nu, \quad \nu=\alpha \xi \mu, \quad \eta=\frac{\alpha}{2}\left(\xi^{2}-\mu^{2}\right) .
$$

The integration of the equation for $a_{-}$is straightforward, and it gives

$$
a_{-}=M_{-} \operatorname{sech}^{2} \theta, \quad M_{-}=\frac{\mu^{2}}{\nu^{2}}\left[\frac{u_{0}}{2}\left(\left|\beta_{1}\right|-\left|\gamma_{1}\right|\right) N+u_{0}^{2}\left(\left|\beta_{1}\right| \beta_{3}-\left|\gamma_{1}\right| \gamma_{3}\right) M\right] .
$$

\section{Conclusions and remarks}

In this paper, an investigation on the interaction between two bright solitons (anomalous dispersion) and one dark soliton (normal dispersion) has been carried out. The problem has been reduced to study a two-component one-dimensional Zakharov-Yajima-Oikawa system in the case of long wave-short wave resonance, by using the multiple scales analysis. The system corresponding to the bright solitons has been discussed further using a Madelung fluid description. In particular, periodic solutions expressed through Jacobi elliptic functions and stationary solutions obtained when $k^{2}=1$ have been presented in two simplifying conditions, namely for constant velocity and for motion with stationary profile.

Remarkably, the above two-component Zakharov-Yajima-Oikawa system is completely integrable and multi-solitons solutions can be found using different methods, as the bi-linear method of Hirota [24]. However, the Madelung fluid description seems to be useful to find various solutions of a generalized ZYO system, containing additional nonlinear terms.

Additionally, the case of two dark and one bright solitons has been also discussed. Here, the use of the multiple scales analysis has allowed us to reduced our problem to a simple onecomponent Zakharov-Yajima-Oikawa system in the resonance condition. In particular, the one-soliton solution has been found. 
Finally, we would like to point out that the discussion for arbitrary values of the parameters is still open and investigations in this direction are in progress. Also numerical solutions of the dynamical model of three interacting waves, discussed in the present paper, could reveal several new aspects of the nonlinearity influence on the system behavior.

\section{Acknowledgements}

Support through CNCSIS program IDEI-571/2008 is acknowledged. The authors are indebted to an anonymous referee for drawing their attention to the paper [26].

\section{References}

[1] Kivshar Y., Agrawal G.P., Optical solitons. From fibers to photonic crystals, Academic Press, San Diego, 2003.

[2] Tratnik M.V., Sipe J.E., Bound solitary waves in a birefringent optical fiber, Phys. Rev. A 38 (1988), 2011-2017.

[3] Menyuk C.R., Nonlinear pulse propagation in birefringent optical fiber, IEEE J. Quant. Electron. 23 (1987), $174-176$.

[4] Christiansen P.L., Eilbeck J.C., Enolskii V.Z., Kostov N.A., Quasi-periodic solutions of the coupled nonlinear Schrödinger equations, Proc. Roy. Soc. London Ser. A 451 (1995), 685-700, hep-th/9410102.

[5] Carretero-Gonzales R., Frantzeskakis D.J., Kevrekidis P.G., Nonlinear waves in Bose-Einstein condensates: physical relevance and mathematical techniques, Nonlinearity 21 (2008), R139-R202, arXiv:0805.0761.

[6] Perez-Garcia V.M., Berloff N.G., Kevrekidis P.G., Konotop V.V., Malomed B.A., Nonlinear phenomena in degenerate quantum gas, Phys. D 238 (2009), 1289-1298.

[7] Agrawal G.P., Nonlinear fiber optics, 2nd ed., Academic Press, New York, 1995.

[8] Akhmediev N., Ankiewicz A., Solitons: nonlinear pulses and beams, Champan and Hall, London, 1997.

[9] Hasegawa A., Kodama Y., Solitons in optical communications, Oxford University Press, 1995.

[10] Onorato M., Osborne A.R., Serio M., Modulational instability in crossing sea states: a possible mechanism, Phys. Rev. Lett. 96 (2006), 014503, 4 pages.

[11] Kivshar Y., Stable vector solitons composed of bright and dark pulses, Optics Lett. 17 (1992), $1322-1325$.

[12] Zakharov V.E., Collapse of Langmuir waves, Zh. Eksp. Teor. Fiz. 62 (1972), 1745-1751 (English transl.: Sov. Phys. JETP 35 (1972), 908-914).

[13] Yajima N., Oikawa M., Formation and interaction of sonic-Langmuir solitons-inverse scattering method, Progr. Theor. Phys. 56 (1976), 1719-1739.

[14] Laedke E.W., Spatschek K.H., Liapunov stability of generalized Langmuir solitons, Phys. Fluids 23 (1980), $44-51$.

[15] Grimshaw R.H.J., Modulation of an internal gravity-wave packet and resonance with mean motion, Studies in Appl. Math. 56 (1977), 241-266.

[16] Benney D.J., General theory for interactions between short and long waves, Studies in Appl. Math. 56 (1977), 81-94.

[17] Djordjevic V.D., Redekopp L.G., 2-dimensional packets of capillary-gravity waves, J. Fluid. Mech. 79 (1977), 703-714.

[18] Davydov A.S., Solitons in molecular systems, D. Reidel Publishing Co., Dordrecht, 1985.

[19] Dodd R.K., Eilbeck J.C., Gibbon J.D., Morris H.C., Solitons and nonlinear wave equations, Academic Press, Inc., London - New York, 1982.

[20] Boiti M., Leon J., Pempinelli F., Spire A., Resonant two-wave interaction in the Davydov model, J. Phys. A: Math. Gen. 37 (2004), 4243-4265.

[21] Visinescu A., Grecu D., Modulational instability and multiple scales analysis of Davydov's model, in Proceedings of Fifth International Conference "Symmetry in Nonlinear Mathematical Physics" (June 23-29, 2003, Kyiv), Editors A.G. Nikitin, V.M. Boyko, R.O. Popovych and I.A. Yehorchenko, Proceedings of Institute of Mathematics, Kyiv $\mathbf{5 0}$ (2004), 1502-1509. 
[22] Radha R., Senthil Kumar C., Lakshmanan M., Tang X.Y., Lou S.Y., Periodic and localized solutions of long wave-short wave resonance interaction equation, J. Phys. A: Math. Gen. 38 (2005), 9649-9663, nlin.SI/0604039.

[23] Yurova M., Application of dressing method for long wave-short wave resonance interaction equation, J. Math. Phys. 48 (2007), 053516, 7 pages, nlin.SI/0612041.

[24] Ohta Y., Maruno K., Oikawa M., Two-component analogue of two-dimensional long wave-short wave resonance interaction equations: a derivation and solutions, J. Phys. A: Math. Theor. 40 (2007), 7659-7672, nlin.SI/0702051.

Maruno K., Ohta Y., Oikawa M., Note for two-component analogue of two-dimensional long wave-short wave resonance interaction system, Glasg. Math. J. 51 (2009), 129-135, arXiv:0812.4591.

[25] Grecu A.T., Grecu D., Visinescu A., Nonlinear dynamics of a Davydov's model with two independent excitonic modes in complex one-dimensional molecular systems, Romanian J. Phys., to appear.

[26] Myrzakulov R., Pashaev O.K., Kholmorodov Kh.T., Particle-like excitations in many component magnonphonon systems, Phys. Scr. 33 (1986), 378-384.

[27] Whitham G.B., Linear and nonlinear waves, John Wiley \& Sons, Inc., New York, 1999.

[28] Karpman V.I., Kruskal E.M., Modulated waves in nonlinear dispersive media, Zh. Eksp. Teor. Fiz. 55 (1968), 530-534 (English transl.: Sov. Phys. JETP 28 (1969), 277-281).

[29] Karpman V.I., Nonlinear waves in dispersive media, International Series of Monographs in Natural Philosophy, Vol. 71, Pergamon Press, Oxford - New York - Toronto, Ont., 1975.

[30] Madelung E., Quantentheorie in hydrodynamischer Form, Z. Physik 40 (1926), 332-326.

[31] Fedele R., Envelope solitons versus solitons, Phys. Scr. 65 (2002), 502-508.

Fedele R., Schamel H., Solitary waves in the Madelung's fluid: connection between the nonlinear Schrödinger equation and the Korteweg-de Vries equation, Eur. Phys. J. B 27 (2002), 313-320.

[32] Manakov S.V., On the theory of two-dimensional stationary self focussing of electromagnetic waves, $Z h$. Eksp. Teor. Fiz. 65 (1973), 505-515 (English transl.: Sov. Phys. JETP 38 (1974), 248-258).

[33] Grecu D., Visinescu A., Fedele R., De Nicola S., Periodic and stationary wave solutions of coupled NLS equations, Romanian J. Phys. 55 (2010), 585-600.

[34] Visinescu A., Grecu D., Fedele R., De Nicola S., Madelung fluid description of the generalized derivative nonlinear Schrödinger equation: special solutions and their stability, Teoret. Mat. Fiz. 160 (2009), 229-239 (English transl.: Theoret. and Math. Phys. 160 (2009), 1066-1074).

[35] Byrd P.F., Friedman M.D., Handbook of elliptic integrals for engineering and scientists, 2nd ed., Die Grundlehren der mathematischen Wissenschaften, Band 67, Springer-Verlag, New York - Heidelberg, 1971. 